\title{
Perceived or Not Perceived: Film Character Models for Expressive NLG
}

\author{
Marilyn A. Walker, Ricky Grant, Jennifer Sawyer, Grace I. Lin, Noah Wardrip-Fruin, \\ and Michael Buell \\ Natural Language and Dialogue Systems Lab \\ University of California Santa Cruz, Santa Cruz, Ca. 95064, USA
}

\begin{abstract}
This paper presents a method for learning models of character linguistic style from a corpus of film dialogues and tests the method in a perceptual experiment. We apply our method in the context of SpyFeet, a prototype role playing game. In previous work, we used the PERSONAGE engine to produce restaurant recommendations that varied according to the speaker's personality $[14,12]$. Here we show for the first time that: (1) our expressive generation engine can operate on content from the story structures of an RPG; (2) PERSONAGE parameter models can be learned from film dialogue; (3) PERSONAGE rule-based models for extraversion and neuroticism are be perceived as intended in a new domain (SpyFeet character utterances); and (4) that the parameter models learned from film dialogue are generally perceived as being similar to the character that the model is based on. This is the first step of our long term goal to create off-theshelf tools to support authors in the creation of interesting dramatic characters and dialogue partners, for a broad range of types of interactive stories and role playing games.
\end{abstract}

Keywords: story dialog, statistical natural language generation, character design

\section{Introduction}

Stories are told through plot structure and narrative, but also critically through dialogue - what a character says, how he says it, and how he reacts to what other characters say. It is widely agreed that progress in interactive story and narrative systems is being hampered by the current approach to dialogue creation, which relies on an individual practitioner's expertise in the creative writing of dialog, often written and rewritten many times $[8,16,15]$. This places a hard limit on the underlying system states that can be expressed [25]. Moreover, the problem is exacerbated when authoring stories that the user is intended to experience many times, with different story trajectories depending on the user's choices and history. It has been suggested that natural language generation techniques offer a promise route to overcoming the dialogue authoring bottleneck for interactive stories and games [25], but surprisingly little work has been done on language generation for story dialogue, as opposed to generating narrative descriptions $[2$, $20,3,4,1]$.

Writers commonly identify two primary challenges in dialogue writing [22]. One is the challenge of revealing subtext. Good dialogue does not explicitly state character personality (e.g., I am a friendly person), character emotional state (e.g., I'm feeling 
hesitant), or character motivation (e.g., I intend to flatter you). Rather, the most important message in most good dialogue appears as subtext, either dramatized or established indirectly by what characters actually say. The second challenge is determining how characters say what they actually say, often referred to as "finding the voice" of each character. Professional writers have developed a number of practices - such as eavesdropping in public, tape recording themselves acting the part, or creating meticulously researched character backgrounds - to help them find character voices.

This paper tests an approach to automatically creating "character voices" based on a corpus-based statistical expressive language generation engine that is trained on the IMSDb corpus of film screen plays [11]. These automatically created character voices are also intended to reveal subtext about character personality and emotion. Our method consists of three components: (1) learning models of character linguistic style from film dialogue screen plays, e.g. the dialogue in Figure 1 from Quentin Tarantino's Pulp Fiction; (2) using the learned models to control the parameters of PERSONAGE, an expressive language generation engine [12]; and (3) experiments on human perceptions of the character utterances created using these models. We test our approach in the context of our prototype role playing game SpyFeet $[18,19]$, a game intended to support dynamic quest selection and dialogue generation, determined by user choices and user relationships with game characters [21].

We believe this sort of corpus-based approach is a much stronger first step than, for example, asking authors to directly tune the parameters of a natural language generation engine. The expertise required to understand the parameters involved, and their interactions, is far removed from the expertise of creative writing - while authors are quite accustomed to presenting character voices through examples, or describing a character's voice a similar to another's (or a blend of familiar voices). Further, being able to explore a landscape of utterances produced through examples could also prove a powerful tool for novice (or even expert) authors who are considering possibilities for character voices. Our initial results, described here, demonstrate that an approach of this sort can produce significant and recognizable variations in linguistic style, even using corpora as small as the utterances of a single character in a screenplay.

Section 2 explains how we use a corpus of film screen plays to learn models of the linguistic style of film characters. Section 3 presents our experimental design, where we first establish human perceptions of the personality of film characters, and then test perceptions of the personality of utterances generated using both learned character models and Big Five personality models. Section 4 presents our experimental results. In previous work, we used the PERSONAGE engine to produce restaurant recommendations that varied according to the speaker's personality, where personality was defined using the Big Five theory of personality [14, 12]. Here we show for the first time that: (1) our expressive generation engine can operate on content from the story structures of an RPG; (2) Personage parameter models can be learned from film dialogue; (3) Personage rule-based models for extraversion and neuroticism are be perceived as intended in a new domain (SpyFeet character utterances); and (4) that the parameter models learned from film dialogue are generally perceived as being similar to the character that the model is based on. Section 5 summarizes the paper.

\section{Learning Character Models}

Procedurally generating interesting dialogue requires a large number of parameters for manipulating linguistic behavior. Our general idea is to develop corpus-based statisti- 
SCENE: JACKRABBIT SLIM S, AFTER FOOD HAS ARRIVED

VINCENT: What do you think about what happened to Antwan?

MIA: Who's Antwan?

VINCENT: Tony Rocky Horror.

MIA: He fell out of a window.

VINCENT: That's one way to say it. Another way is, he was thrown out. Another was is, he was thrown out by Marsellus.

And even another way is, he was thrown out of a window by Marsellus because of you.

MIA: Is that a fact?

VINCENT: No it's not, it's just what I heard.

MIA: Who told you this?

VINCENT: They.

Mia and Vincent smile.

MIA: They talk a lot, don't they?

VINCENT: They certainly do.

MIA: Well don't be shy Vincent, what exactly did they say?

Vincent is slow to answer.

MIA: Let me help you Bashful, did it involve the F-word?

VINCENT: No. They just said Rocky Horror gave you a foot massage.

MIA: And...?

VINCENT: No and, that's it.

VINCENT: No and, that's it.
MIA: You heard Marsellus threw Rocky Horror out of a four-story window because he massaged my feet?

VINCENT: Yeah.
MIA: And you believed that?

MIA: And you believed that?
VINCENT: At the time I was told, it seemed reasonable.

MIA: Marsellus throwing Tony out of a four story window for giving me a foot massage seemed reasonable?

VINCENT: No, it seemed excessive. But that doesn't mean it didn't happen. I heard Marsellus is very protective of you.

MIA: A husband being protective of his wife is one thing. A husband almost killing another man for touching his wife' feet is something else.

VINCENT: But did it happen?

MIA: The only thing Antwan ever touched of mine was my hand, when he shook it. I met Anwan once at my wedding then never again. The truth is, nobody knows why Marsellus tossed Tony Rocky Horror out of that window except Marsellus and Tony Rocky Horror. But when you scamps get together, you're worse than a sewing circle.

Fig. 1. Scene from Pulp Fiction.

cal models of character linguistic style by counting linguistic reflexes (features) in film dialogue, and then use these models to control the parameters of the PERSONAGE generator $[14,12]$. The PERSONAGE parameters that we wish to control with our character models are shown in Table 1.

Corpus and Features. Our corpus consists of 862 film scripts from the IMSDb website, representing 7400 characters, with a total of 664000 lines of dialogue and 9599000 tokens. Our snapshot of IMSDb is from May 19, 2010. We use the IMDB ontology to define groupings of character types according to the following attributes: GENRE, DIRECTOR, and YEAR. Note that most films belong to multiple genres. For example, Pulp Fiction belongs to crime, drama, and thriller. This allows for characters to be grouped in multiple categories. We hand-annotated CHARACTER GENDER because we thought that gender might be a factor in defining linguistic styles [9].

The linguistic reflexes (features) that we count in the screenplays are based on previous studies of features useful as indicators of a person's personality, gender or social class $[13,6,17,9]$. Table 2 enumerates our feature sets. For most of the features, there is a particular parameter in PERSONAGE (in Table 1) whose parameter value should be affected by that feature's presence or absence in a character's dialogic utterances. The Basic features capture aspects of style such as how much a character talks and how many words they use (the VERBOSITY parameter). The Dialogue Act features are based on a dialogue act tagger trained on the NPS Chat Corpus 1.0 [5]. The First Dialogue Act is the Dialogue Act of the first sentence of each turn. Several dialogue act features indicate the use of the parameters INITIAL REJECTION or ACKNOWLEDGMENT. Others we do not currently utilize. Pragmatic Markers include both categories of pragmatic markers and individual word count/ratio. Pragmatic marker features indicate which aggregation operations to use such as JUSTIFY-CUE WORD (See Table 1) or which pragmatic markers to insert, such as EMPHASIZERS or SOFTENER HEDGES. The Merge Ratio uses a 


\begin{tabular}{|c|c|}
\hline Parameter & Description \\
\hline \multicolumn{2}{|r|}{ Content Planning } \\
\hline VERBOSITY & Control the number of propositions in the utterance \\
\hline REPETITIONS & Repeat an existing proposition \\
\hline CONTENT POLARITY & Control the polarity of the propositions expressed, i.e., referring to negative or positive facts \\
\hline $\begin{array}{l}\text { REPETITIONS POLAR- } \\
\text { ITY }\end{array}$ & Control the polarity of the restated propositions \\
\hline CONCESSIONS & Emphasize one attribute over another \\
\hline $\begin{array}{l}\text { CONCESSIONS POLAR- } \\
\text { ITY }\end{array}$ & Determine whether positive or negative attributes are emphasized \\
\hline POLARIZATION & Control whether the expressed polarity is neutral or extreme \\
\hline $\begin{array}{l}\text { Positive CONTENT } \\
\text { FIRST }\end{array}$ & Determine whether positive propositions - including the claim — are uttered first \\
\hline INITIAL REJECTION & Begin the utterance with a mild rejection \\
\hline \multicolumn{2}{|r|}{ Syntactic Template Selection } \\
\hline SELF-REFERENCES & Control the number of first person pronouns \\
\hline SYNTACTIC COMPLEX- & Control the syntactic complexity (syntactic embedding) \\
\hline $\begin{array}{l}\text { ITY } \\
\text { TEMPLATE POLARITY }\end{array}$ & Control the connotation of the claim, i.e., whether positive or negative affect is expressed \\
\hline \multicolumn{2}{|c|}{$\begin{array}{ll} & \text { Aggregation Operations } \\
\end{array}$} \\
\hline PERIOD & Leave two propositions in their own sentences \\
\hline RELATIVE Clause & Aggregate propositions with a relative clause \\
\hline WITH CUE WORD & Aggregate propositions using "with" \\
\hline CONJUNCTION & Join two propositions using a conjunction, or a comma if more than two propositions \\
\hline MERGE & Merge the subject and verb of two propositions \\
\hline ALSO CUE WORD & Join two propositions using "also" \\
\hline CONTRAST-CUE WORD & Contrast two propositions using "while", "but", "however", "on the other hand" \\
\hline JUSTIFY-CUE WORD & Justify a proposition using "because", "since","so" \\
\hline CONCEDE-CUE WORD & Concede a proposition using "although", "even if", "but/though" \\
\hline MERGE WITH COMMA & Restate a proposition by repeating only the object \\
\hline \multicolumn{2}{|r|}{ Pragmatic Markers } \\
\hline STUTTERING & Duplicate the first letters of a restaurant's name \\
\hline PRONOMINALIZATION & Replace occurrences of the restaurant's name by pronouns \\
\hline NEGATION & Negate a verb by replacing its modifier by its antonym \\
\hline SOFTENER HEDGES & Insert syntactic elements to mitigate the strength of a proposition \\
\hline EMPHASIZER HEDGES & Insert syntactic elements to strengthen a proposition \\
\hline ACKNOWLEDGEMENTS & Insert an initial back-channel \\
\hline FILLED PAUSES & Insert syntactic elements expressing hesitancy \\
\hline EXCLAMATION & Insert an exclamation mark \\
\hline EXPLETIVES & Insert a swear word \\
\hline NEAR EXPLETIVES & Insert a near-swear word \\
\hline TAG QUESTION & Insert a tag question \\
\hline IN-GROUP MARKER & Refer to the hearer as a member of the same social group \\
\hline
\end{tabular}
Lexical Choice

LEXICON FREQUENCY Control the average frequency of use of each content word, according to BNC frequency counts

LEXICON WORD Control the average number of letters of each content word

\begin{tabular}{l|l}
$\begin{array}{l}\text { LENGTH } \\
\text { VERB STRENGTH }\end{array}$ & Control the strength of the verbs \\
\hline
\end{tabular}

Table 1. PERSONAGE's generation parameters

\begin{tabular}{|c|c|}
\hline Feature & Description (Label) \\
\hline Basic & $\begin{array}{l}\text { number of sentences (NumSents), sentences per turn (NumSentsPerTurn), } \\
\text { number of verbs (NumVB), number of verbs per sentence (VBPerSents) }\end{array}$ \\
\hline Polarity & overall polarity (polarity-overall), polarity of sentences (polarity-sents) \\
\hline Dialogue Act (DA) & $\begin{array}{l}\text { Accept, Bye, Clarify, Continuer, Emotion, Emphasis, Greet, No-Answer, Re- } \\
\text { ject, Statement, Wh-Question, Yes-Answer, Yes-No-Question, Other }\end{array}$ \\
\hline First DA & Same as DA but only look at first sentence of each turn \\
\hline Merge Ratio & merging of subject and verb of two propositions (merge-ratio) \\
\hline Passive Sentence Ratio & passive sentence count (passive-ratio) \\
\hline Concession polarity & polarity for concessions (concess-polarity) \\
\hline LIWC Word Categories & Each prefixed as LIWC- \\
\hline Pragmatic Markers & $\begin{array}{l}\text { wc-taboo, wc-seq, wc-opinion, wc-aggregation, wc-softeners, wc-emphatics, } \\
\text { wc-ack, wc-pauses, wc-concession, wc-concede, wc-justify, wc-contrast, wc- } \\
\text { conjunction, wc-ingroup, wc-near_swear, wc-relative }\end{array}$ \\
\hline Tag Question Ratio & tag question ratio (tag-ratio) \\
\hline Word Length & average content word length (avg-content-wlen) \\
\hline Verb Strength & 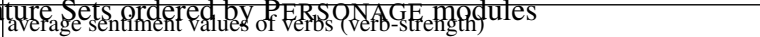 \\
\hline
\end{tabular}


grammar operating on POS labels that looks for verb+noun+conjunction+noun. The Passive Sentence Ratio uses scripts from http://code.google.com/p/narorumo, under source/browse/trunk/passive to detect passive sentences. These scripts implement the rule that if a to-be verb is followed by a non-gerund, the sentence is probably in passive voice. The Concession Polarity feature is based on finding the polarity for the concession in a sentence if it exists, using the Polarity feature set. The LIWC tool provides a lexical hierarchy that counts the use of different types of words, including cue-words, emotion words, and pronouns inter alia. These are used to map to both aggregation operations and pragmatic markers. The Tag Question Ratio is also based on a set of regular expressions. The features Word Length and Verb Strength control the lexical choice parameters. Word Length first uses WordNet tags to find content words (noun, adjective, adverb, and verb), and then takes the mean of their length in characters. Verb Strength is the mean sentiment scores of all verbs. Lexical frequency is approximated from a combination of the features LIWC-6LTR and word length.

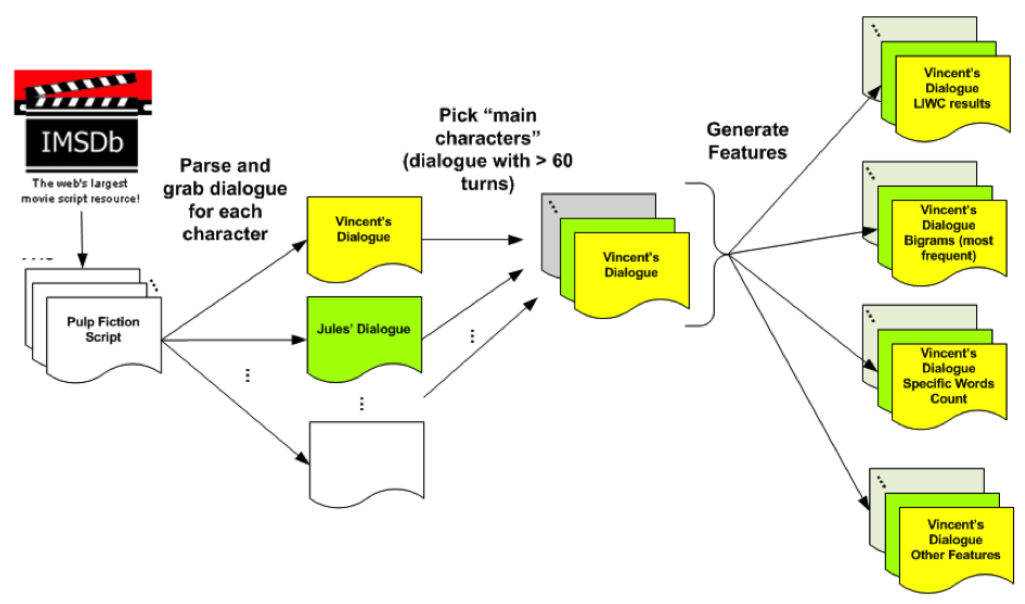

Fig. 2. Method

Method. Fig. 2 shows the flow of our experiment. In sum, our method is:

1. Collect movie scripts from The Internet Movie Script Database (IMSDb).

2. Parse each movie script to extract dialogic utterances, producing an output file containing utterances of exactly one character of each movie (e.g., pulp-fictionvincent.txt has all of the lines of the character Vincent).

3. Select characters from those with more than 60 turns of dialogue.

4. Extract features representing the linguistic behaviors of each character.

5. Learn models of character linguistic styles based on these features.

6. Use character models to control parameters of the PERSONAGE generator.

7. Evaluate human perceptions of dialogic utterances generated using the character models.

Character Models. Sample character models derived from the procedure above are provided in Table 3 . Table 4 illustrates the result of applying these models of character 


\begin{tabular}{|c|c|c|c|c|c|c|}
\hline Parameter & Alvy & Annie & ndy & arion & Mia & incent \\
\hline Co & ent $P$ & annin & & & & \\
\hline Verbosity & .79 & .78 & .36 & .65 & .49 & .18 \\
\hline Repetitions & .38 & 0 & 0 & 0 & .28 & .51 \\
\hline Content Polarity & .09 & .77 & .15 & .15 & .15 & .50 \\
\hline Polarization & .39 & .72 & .22 & .21 & .22 & .57 \\
\hline Repetitions Polarity & .54 & .79 & .29 & .29 & .29 & .64 \\
\hline Concessions & .83 & .83 & .83 & .89 & .89 & .58 \\
\hline Concessions Polarity & .56 & .26 & .56 & .26 & .26 & .49 \\
\hline Positive Content First & 0 & 1.00 & 0 & 0 & 0 & 1.00 \\
\hline Initial Rejection & 0 & 0 & 0 & 0 & 0 & 0 \\
\hline Syntacti & Temp & te Se & ectio & & & \\
\hline Use of First Person in Claim & .39 & .6 & .39 & .39 & .39 & .54 \\
\hline Claim Polarity & .57 & .57 & .57 & .49 & .56 & .50 \\
\hline Claim Complexity & .71 & .31 & .47 & .15 & .56 & .56 \\
\hline Aggre & tion & perat & ions & & & \\
\hline Period & .05 & .04 & .24 & .04 & .24 & 0 \\
\hline Relative Clause & 0 & 0 & .95 & .97 & .53 & .3 \\
\hline With cue word & .44 & .51 & .05 & .34 & .31 & .25 \\
\hline Conjunction & .30 & .21 & .22 & .18 & .08 & 0 \\
\hline Merge & .61 & .87 & .83 & .65 & .59 & .77 \\
\hline Also cue Word & .12 & .05 & .05 & .05 & .07 & .05 \\
\hline Contrast-Cue word & .76 & .85 & 0 & .84 & .76 & .96 \\
\hline Justify-Cue Word & .97 & .48 & 0 & .61 & .61 & .45 \\
\hline Concede-Cue Word & 1.00 & 0 & 0 & 1.00 & 0 & .25 \\
\hline Merge With Comma & .27 & .42 & .5 & .5 & .32 & .5 \\
\hline Pras & natic & Tarke & & & & \\
\hline Stuttering & .54 & .54 & .04 & .04 & .54 & .09 \\
\hline Pronominalization & 1.00 & 1.00 & 1.00 & .75 & .5 & 1.00 \\
\hline Negation & 0 & 0 & 0 & 0 & 0 & 0 \\
\hline Softener Hedges & 1.00 & 1.00 & 0 & 1.00 & 0 & 0 \\
\hline Emphasizer hedges & 0 & 1.0 & 0 & & 1.00 & 0 \\
\hline Acknowledgements & 1.00 & 1.00 & 0 & 0 & 1.00 & 0 \\
\hline Filled Pauses & 1.00 & 1.00 & 0 & 0 & 0 & 0 \\
\hline Exclamation & 0 & 0 & 0 & 1.00 & 0 & 1.00 \\
\hline Expletives & 0 & 0 & 0 & 0 & 0 & 1.00 \\
\hline Near Expletives & 0 & 0 & 0 & 0 & 0 & 0 \\
\hline Tag Question & 1.00 & 1.00 & 0 & & 1.00 & 0 \\
\hline In-Group Marker & 0 & 0 & 0 & 1.00 & 0 & 0 \\
\hline & xical & hoice & & & & \\
\hline Lexicon Frec & .19 & .19 & .28 & .19 & .55 & .18 \\
\hline Lexicon Word Length & .21 & .13 & .21 & .13 & .78 & .28 \\
\hline Verb Strength & .59 & .59 & .5 & .61 & .5 & .49 \\
\hline
\end{tabular}

Table 3. Sample Learned Character Models

to SpyFeet utterances, and shows some of the variation that we are currently able to produce.

There are many different ways we could learn such models [10, 23, 24]. Here, we estimate models using vectors of features representing individual characters, and then derive distinctive features for that character by normalizing these feature counts against a representative population. For each feature $x_{i}$, the normalized value $z_{i}$ is calculated as:

$$
\frac{x_{i}-\overline{x_{i}}}{\sigma_{x i}}
$$




\begin{tabular}{|l|l|l|}
\hline Film & Character & Generated Utterances \\
\hline \hline Annie Hall & Alvy & $\begin{array}{l}\text { I don't know. People say Cartmill is st-strange, alright? Err... on the other hand, Idon't rush } \\
\text { to judgment. } \\
\text { Right, I am not sure, would you be? I will tell something you because you br-brought me } \\
\text { cabbage. } \\
\text { Oh I am not sure. Wolf wears a hard shell. On the other hand, he is ge-ge-gentle, isn't he? } \\
\text { I see, I don't know. I respect Wolf, wouldn't you? He, however, isn't my close friend. } \\
\text { Yeah, I don't know. Sparrow conveys excitement to my life, so I am fr-fr-friends with her. }\end{array}$ \\
\hline $\begin{array}{l}\text { Indiana } \\
\text { I don't rush to judgment, but people say Cartmill is strange. }\end{array}$ & $\begin{array}{l}\text { I will tell something you since you brought me cabbage. } \\
\text { Wolf is gentle but he wears a hard shell. } \\
\text { Wolf isn't my close friend. But I respect him. } \\
\text { I am friends with Sparrow since she brings excitement to my life. }\end{array}$ \\
\hline $\begin{array}{l}\text { Annie Hall } \\
\text { Indy }\end{array}$ & $\begin{array}{l}\text { Annie } \\
\text { judgment. I don't know, do you? People say Cartmill is strange while I don't rush to um.. } \\
\text { I don't know. I think that you brought me cabbage, so I will tell something to you, alright? } \\
\text { Yeah, I am not sure, would you be? Wolf wears a hard shell but he is really gentle. } \\
\text { I see, I am not sure. Obviously, I respect Wolf. However, he isn't my close friend, is he? } \\
\text { Come on, I am not sure. Because Sparrow brings excitement to my life, I am friends with } \\
\text { her, you see? }\end{array}$ \\
$\begin{array}{l}\text { Basically, I don't rush to judgment. On the other hand, people say Cartmill is strange, he is } \\
\text { strange. } \\
\text { Yeah, I can answer since you brought me cabbage that. } \\
\text { Everybody knows that Wolf wears a hard shell. He, however, is gentle. } \\
\text { I respect Wolf. However, he isn't my damn close friend. } \\
\text { Oh God I am friends with Sparrow because she brings excitement to my life. }\end{array}$ \\
\hline $\begin{array}{l}\text { Pulp } \\
\text { Fion }\end{array}$
\end{tabular}

Table 4. Utterances for SpyFeet generated using Film Character Models

There is a choice about the population of characters used for the normalization, i.e. which set of characters are used to calculate the mean $\overline{x_{i}}$ and the standard deviation $\sigma_{x i}$. For example, for a female character, obvious choices include all the characters, all the female characters, or all the female action characters. Here we normalize individual characters against all of the characters of the same gender. Any z-score greater than 1 or less than -1 is more than one standard deviation away from the mean. Z-scores greater and less than +-1.96 indicate significant differences of the use of that linguistic feature by that character compared to other characters. However for experimental purposes we map any z-score greater than 1 or less than -1 into one or more PERSONAGE generation parameters. There are three different ways in which our mappings of feature counts to parameters could be incomplete:

1. We have a parameter in PERSONAGE but none of the features we are counting are good indicators that we should use it (e.g., Competence Mitigation);

2. We have a feature we are counting but no parameter to map it to (e.g., LIWCDiscrep);

3. There is some aspect of linguistic style that is essential to expressing a particular character's style, but we currently do not have a feature that indicates when a character has that style, nor do we have any existing parameter that could manifest that linguistic reflex.

\section{Experimental Setup}

Our goal is to test the character models and mappings as described above. The simplest way to do this is to ask human participants to rate a set of utterances produced using different models in terms of their similiarty of linguistic style to the mimicked character. However our concern was that a single linguistic cue could act as a "give-away" for the 
intended character. For example, if the Annie character from Woody Allen's Annie Hall uses tag-questions (among other linguistic style differentiators), perhaps the use of tagquestions in a test utterance would cue a human participant that the test utterance was intended by the experimenters to mimic Annie.

Therefore, we designed an experiment to first indirectly test the similarity in perceived personality of, e.g. the Annie character as written in the film, to the personality of utterances of SpyFeet characters produced using an Annie model of linguistic style. Our experimental method consists of three phases each intended to establish human perceptions of different aspects of utterances generated using character models. In Phase I, we select 3 scenes from each of the original films, illustrating the utterance styles of 6 characters ( 3 male and 3 female). We collect user perceptions of the personality of those characters using the Ten Item Personality Inventory (TIPI) [7, 12]. In Phase II, using the PERSONAGE generator, we generate dialogic utterances for the characters in the story of the SpyFeet RPG, using both (1) the film character model; and (2) 6 rulebased personality models from our previous work (high and low values for extraversion, neuroticism and agreeableness) [12]. We collect user perceptions of the personality of SpyFeet characters whose linguistic style is controlled by these models (6 film character models and 6 personality models), again using the TIPI [7]. In Phase III, using all the utterances generated in Phase II, for each film character model, we generate a page showing the user (1) the three scenes for each character (from Phase I); and (2) all of the generated utterances using all of the film character models and all of the rule-based personality models. Then we ask users to judge on a scale of $1 \ldots 7$ how similar the generated utterance is to the style of the film character as illustrated in the three scenes. Users are instructed to use the whole scale, and thus effectively rank the generated utterances for similarity to the film character. Each phase supports different analyses of the user perceptions of SpyFeet characters. Using the data collected in Phase I, we establish user perceptions of film characters on the Big Five personality traits of extraversion, neuroticism and agreeableness. Then using the data from Phases I and II, we examine the correlations between user perceptions of the film character's original utterances (Annie, Alvy, Vincent, Mia, Indiana, Marion) and SpyFeet utterances that were generated using the learned models of the film character. Our Hypotheses are:

- H1: The rule-based models for personality expression (previously tested in the restaurant recommendation domain), will be perceived as expressing that personality in the SpyFeet story domain (Phase II).

- H2: Utterances generated using character models (Phase II) will correlate more strongly with character personality perceptions than utterances generated using rule-based models for personality expression (also Phase II).

- H3: Utterances generated using character models will be perceived as being more similar to that character than utterances generated using another randomly selected character model (Phase III).

\section{Experimental Results}

29 subjects ( 13 female and 16 male, ages ranging from 22 to 44) participated in a webbased experiment.

Phase I. We made no predictions about the results in Phase I. Our goal was to establish personality judgements for the six characters and test whether, in terms of Big Five traits the characters are perceived as having distinctive personalities. Table 5 shows the 
mean values of the TIPI scale judgements for Big Five traits of Extraversion, Emotional Stability and Agreeableness for the six characters.

\begin{tabular}{|l|c|c|c|c|c|c|}
\hline & \multicolumn{5}{|c|}{ Character } \\
Trait & Alvy & Annie & Indy & Marion & Mia & Vincent \\
\hline Extraversion & 2.8 & 4.4 & 4.2 & 5.5 & 4.8 & 4.6 \\
Emotional Stability & 2.0 & 2.5 & 5.0 & 3.8 & 4.4 & 4.1 \\
Agreeableness & 4.0 & 4.5 & 3.3 & 3.9 & 4.0 & 4.1 \\
\hline
\end{tabular}

Table 5. Big Five Personality Scores for Film Character Original Utterances

We combined the personality judgments for a character for all three Big Five traits into a single vector and computed paired t-tests (two-tailed) on these vectors to determine whether characters were perceived as having distinct personalities (within subjects). The results indicate that the personality of Alvy is perceived as being significantly different from all of the other characters ( $\mathrm{df}=90,3.3<\mathrm{t}<7.4, \mathrm{p}<.001$ ). However Indy is only different from Alvy $(\mathrm{df}=90, \mathrm{t}=4.8, \mathrm{p}<.0001)$. Marion is only different from Alvy and Annie ( $\mathrm{df}=90,3.3<\mathrm{t}<7.4, \mathrm{p}<.001$ ), Vincent is only different from Alvy and Annie $(\mathrm{df}=90,2.3<\mathrm{t}<6.6, \mathrm{p}<.02)$, and Mia is only different from Alvy and Annie ( $\mathrm{df}=90,3.1<\mathrm{t}<7.4, \mathrm{p}<.003)$. These results suggest that the differences in perceived personality across different characters are small, with the Tarantino and Spielberg characters being perceived as having similar personalities. However as we show below in Phase III, there are distinctive differences in their linguistic styles that are perceivable.

Phase II. Our predictions (Hypotheses H1 and H2) were that rule-based models for personality expression will be perceived as expressing that personality in the SpyFeet story domain, and that utterances generated using character models will correlate more strongly with character personality perceptions than utterances generated using rulebased models for personality expression.

To date we have only tested $\mathrm{H} 1$. The final paper will report correlational tests to test Hypothesis 2. Our results for $\mathrm{H} 1$ are mixed. We tested whether utterances generated with high and low extraversion models, high and low agreeableness models and high and low emotional stability models are perceived as expressing those traits. A paired t-test comparing the extraversion ratings of high $(x \equiv 5.2)$ and low extraversion $(x \equiv 3.3)$ utterances showed significant differences $(\mathrm{df}=28, \mathrm{t}=7.7, \mathrm{p}<.0001)$, as did a paired t-test comparing the emotional stability ratings of high $(x \equiv 5.5)$ and low $(x \equiv 2.7)$ emotional stability utterances $(\mathrm{df}=28, \mathrm{t}=10.8, \mathrm{p}<.0001)$. However differences in high ( $x \equiv 3.4$ ) and low ( $x \equiv 3.4$ ) agreeableness were not perceived in the SpyFeet domain, when we used the agreeableness model that had previously been successful in the restaurant recommendation domain $(\mathrm{df}=28, \mathrm{t}=.72, \mathrm{p}=.47 \mathrm{~ns})$. There are several possible reasons for this; perhaps the limited set of utterances tested, as shown in Table 4, do not do a good job of showing the variability in agreeableness that the PERSONAGE generator is capable of, or perhaps manifesting agreeableness in the SpyFeet domain requires the addition of new parameters to PERSONAGE.

Phase III. Our prediction (Hypothesis H3) was that utterances generated using character models would be more similar to that character than utterances generated using another randomly selected character model. Table 6 shows the average similarity score judgments between utterances produced with a particular character model and the ut- 
terances of that character in the original film. For example Row 1 shows the judgments for the similarity of utterances generated with each character model to the utterances of the Alvy character in the original Annie Hall screen play. Similarity scores are scalar values from 1...7. The strongest possible result would be a diagonal matrix with 7's along the diagonal and 0's in all the other cells, i.e. a only utterances generated with a particular character's model would be judged as being at all similar to that character. In general, what we are looking for is a matrix with the highest values along the diagonal.

\begin{tabular}{|l|c|c|c|c|c|c|}
\hline Character & Alvy & Annie & Indy & Marion & Mia & Vincent \\
\hline Alvy & 5.2 & $\mathbf{4 . 2}$ & $\mathbf{2 . 1}$ & $\mathbf{2 . 6}$ & $\mathbf{2 . 8}$ & $\mathbf{2 . 3}$ \\
\hline Annie & 4.2 & 4.3 & $\mathbf{2 . 8}$ & $\mathbf{3 . 4}$ & 3.9 & $\mathbf{2 . 9}$ \\
\hline Indy & $\mathbf{1 . 4}$ & $\mathbf{2 . 2}$ & 4.5 & $\mathbf{2 . 8}$ & $\mathbf{3 . 3}$ & $\mathbf{3 . 8}$ \\
\hline Marion & $\mathbf{1 . 6}$ & $\mathbf{2 . 8}$ & 3.7 & 3.1 & $\mathbf{4 . 1}$ & $\mathbf{4 . 2}$ \\
\hline Mia & $\mathbf{1 . 7}$ & $\mathbf{2 . 4}$ & 4.3 & 3.2 & 3.6 & 4.3 \\
\hline Vincent & $\mathbf{2 . 1}$ & $\mathbf{3 . 2}$ & 4.5 & $\mathbf{3 . 5}$ & $\mathbf{3 . 6}$ & 4.6 \\
\hline
\end{tabular}

Table 6. Mean Similarity Scores between Characters and Character Models. Significant differences between the designated character and each other character are shown in bold.

We conducted paired t-tests comparing the similarity scores of each other character model to the similarity scores for the matching model (e.g. we compared similarity scores for utterances generated using Alvy's model to utterances generated using Indy's model, collected in the context of the user looking at the screenplay for Indiana Jones.).

For Annie Hall, utterances generated using the Alvy model (first row of Table 6) are significantly more similar to Alvy than utterances generated using any other model (df $=28,3.16 ; \mathrm{t} 8.35, \mathrm{p}<.004$ ). The utterances generated using the Annie model (first row of Table 6) are significantly more similar to Annie than utterances generated with the Indy $(\mathrm{df}=28, \mathrm{t}=3.75, \mathrm{p}<.0008)$, Marion $(\mathrm{df}=28, \mathrm{t}=2.08, \mathrm{p}<.05)$, and Vincent $(\mathrm{df}$ $=28, \mathrm{t}=2.90, \mathrm{p}<.007)$, but not different than utterances generated with the models for Alvy $(\mathrm{df}=28, \mathrm{t}=.09, \mathrm{~ns})$, and Mia $(\mathrm{df}=28, \mathrm{t}=.85, \mathrm{~ns})$.

For Indiana Jones, utterances generated using the Indy model (third row of Table 6) are significantly more similar to Indy than utterances generated using any other model $(\mathrm{df}=28,2.67 ; \mathrm{t} ; 7.99, \mathrm{p}<.01)$ Utterances generated using the Marion model (fourth row of Table 6) are also significantly more similar to Marion than utterances generated using Alvy ( $\mathrm{df}=28, \mathrm{t}=4.70, \mathrm{p}<.0001)$, Mia $(\mathrm{df}=28, \mathrm{t}=2.66, \mathrm{p}<.013)$, or Vincent models $(\mathrm{df}=28, \mathrm{t}=3.24, \mathrm{p}<.003)$, but not different than the Annie model $(\mathrm{df}=28, \mathrm{t}$ $=.52, \mathrm{p}=.65 \mathrm{~ns})$ or the Indy model $(\mathrm{df}=28, \mathrm{t}=1.98, \mathrm{p}<.057)$.

For Pulp Fiction, utterances generated using the Mia model (fifth row of Table 6) are significantly more similar to Mia than utterances generated from the Alvy $(\mathrm{df}=28$, $\mathrm{t}=6.72, \mathrm{p}<.0001)$, and Annie $(\mathrm{df}=28, \mathrm{t}=3.24, \mathrm{p}<.003)$ models, but not different than those using models for Indy $(\mathrm{df}=28, \mathrm{t}=1.67, \mathrm{p}=.11 \mathrm{~ns})$, Marion $(\mathrm{df}=28, \mathrm{t}=$ $1.06, \mathrm{p}=.30 \mathrm{~ns})$, and Vincent $(\mathrm{df}=28, \mathrm{t}=1.58, \mathrm{p}=.13 \mathrm{~ns})$. The fact that the model for the Mia character was trained on the fewest number of utterances (she has only 81 lines in the film) could contribute to the lack of perceivable differences. Utterances generated using the Vincent model (sixth row of Table 6) are significantly more similar to Vincent than utterances generated using Alvy $(\mathrm{df}=28, \mathrm{t}=6.59, \mathrm{p}<.0001)$, Annie $(\mathrm{df}=28, \mathrm{t}$ $=3.54, \mathrm{p}<.0014)$, Marion $(\mathrm{df}=28, \mathrm{t}=2.57, \mathrm{p}<.02)$, and Mia models $(\mathrm{df}=28, \mathrm{t}=$ $2.25, \mathrm{p}<.03)$, but not different than the Indy model $(\mathrm{df}=28, \mathrm{t}=.86, \mathrm{p}=.18 \mathrm{~ns})$. 


\section{Discussion}

If deeply interactive stories are to feature dialog, we must move beyond a model of pure hand authoring. As stories vary in terms of the events that take place, the characters that are present, the dynamic states of relationships between characters, and so on, we must be able to dynamically generate dialogue that reflects and drives the state of the fictional world while expressing character in a manner controllable by an author. But asking authors to, for example, specify the parameter settings for a complex natural language generation engine is at odds with the skillsets and approaches of most, whether experts or beginners.

In this paper we have demonstrated the first step toward an alternative approach: developing models of character linguistic style from examples, specifically using character utterances in film scripts. Our results are encouraging, showing that utterances generated in a different domain (that of an outdoor role-playing game) recognizably display important subtext for character personality as well as style that is more similar to the modeled character than to others (though, perhaps unsurprisingly, characters from the same genre or film are often more similar to each other than to others).

After this initial step, much work remains to be done. For example, just as a character's plot actions in an interactive story must be related to the current state of the world and actions of other characters, so must linguistic actions take place in context. Our current model does not represent anything about the relation between dialogic utterances across speakers. The importance of such relations can be seen in Figure 1, in which paraphrastic and echoic aspects of the dialogue actually seem to be an interesting part of Mia's linguistic style - as well as an indication of her character's current stance toward Vincent. This points to another important area for future work, as we explore how character linguistic style varies across situations in order to help communicate emotional dynamics to the audience.

\section{References}

1. André, E., Rist, T., van Mulken, S., Klesen, M., Baldes, S.: The automated design of believable dialogues for animated presentation teams. In: S. Prevost J. Cassell, J.S., Churchill, E. (eds.) Embodied conversational agents, pp. 220-255. MIT Press, Cambridge, MA (2000)

2. Beskow, J., Cerrato, L., Granström, B., House, D., Nordenberg, M., Nordstrand, M., Svanfeldt, G.: Expressive animated agents for affective dialogue systems. In: Proceedings of the Tutorial and Research Workshop on Affective Dialogue Systems (ADS'04). pp. 301-304. Kloster Irsee, Tyskland (2004)

3. Cavazza, M., Charles, F.: Dialogue generation in character-based interactive storytelling. In: AAAI First Annual Artificial Intelligence and Interactive Digital Entertainment Conference, Marina del Rey, California, USA (2005)

4. Elson, D., McKeown, K.: Building a bank of semantically encoded narratives. In: Proceedings of the Seventh International Conference on Language Resources and Evaluation (LREC 2010), Malta. Citeseer (2010)

5. Forsyth, E., Martell, C.: Lexical and discourse analysis of online chat dialog. IEEE Computer Society (2007)

6. Furnham, A.: Language and personality. In: Giles, H., Robinson, W. (eds.) Handbook of Language and Social Psychology. Winley (1990)

7. Gosling, S.D., Rentfrow, P.J., Swann, W.B.: A very brief measure of the big five personality domains. Journal of Research in Personality 37, 504-528 (2003) 
8. Hayes-Roth, B., Brownston, L., Sincoff, E.: Directed improvisation by computer characters. Tech. Rep. KSL 95-04, Knowledge Systems Laboratory, Stanford University (1995)

9. Ireland, M., Pennebaker, J.: Authors' gender predicts their characters' language. In submission (2011)

10. Isard, A., Brockmann, C., Oberlander, J.: Individuality and alignment in generated dialogues. In: Proceedings of the 4th International Natural Language Generation Conference (INLG06). pp. 22-29. Sydney, Australia (2006)

11. Lin, G.I., Walker, M.A.: All the world's a stage: Learning character models from film. In: Proceedings of the Seventh AI and Interactive Digital Entertainment Conference. AIIDE '11, AAAI (2011)

12. Mairesse, F., Walker, M.: Towards personality-based user adaptation: psychologically informed stylistic language generation. User Modeling and User-Adapted Interaction pp. 1-52 (2010)

13. Mairesse, F., Walker, M.A., Mehl, M.R., Moore, R.K.: Using linguistic cues for the automatic recognition of personality in conversation and text. Journal of Artificial Intelligence Research (JAIR) 30, 457-500 (2007)

14. Mairesse, F., Walker, M.A.: Controlling user perceptions of linguistic style: Trainable generation of personality traits. Computational Linguistics (2011)

15. Mateas, M.: The authoring bottleneck in creating AI-based interactive stories. In: Proceedings of the AAAI 2007 Fall Symposium on Intelligent Narrative Technologies (2007)

16. Mateas, M., Stern, A.: Façade: An experiment in building a fully-realized interactive drama. In: Proceedings of the Game Developers Conference, Game Design track (2003)

17. Pennebaker, J.W., King, L.A.: Linguistic styles: Language use as an individual difference. Journal of Personality and Social Psychology 77, 1296-1312 (1999)

18. Reed, A., Samuel, B., Sullivan, A., Grant, R., Grow, A., Lazaro, J., Mahal, J., Kurniawan, S., Walker, M., Wardrip-Fruin, N.: Spyfeet: An exercise rpg. In: Proceedings of the Sixth International Conference on the Foundations of Digital Games. pp. 310-312. FDG '11, ACM, New York, NY, USA (2011)

19. Reed, A., Samuel, B., Sullivan, A., Grant, R., Grow, A., Lazaro, J., Mahal, J., Kurniawan, S., Walker, M., Wardrip-Fruin, N.: A step towards the future of role-playing games: The spyfeet mobile rpg project. In: Proceedings of the Seventh AI and Interactive Digital Entertainment Conference. AIIDE '11, AAAI (2011)

20. Rowe, J., Ha, E., Lester, J.: Archetype-Driven Character Dialogue Generation for Interactive Narrative. In: Intelligent Virtual Agents. pp. 45-58. Springer (2008)

21. Sullivan, A., Mateas, M., Wardrip-Fruin, N.: Rules of engagement: moving beyond combatbased quests. In: Proceedings of the Intelligent Narrative Technologies III Workshop. pp. 1-8. ACM (2010)

22. Trottier, D.: The Screenwriter's Bible: A Complete Guide to Writing, Formatting, and Selling Your Script. Silman-James Press, 5th edn. (2010)

23. Walker, M., Rambow, O., Rogati, M.: Training a sentence planner for spoken dialogue using boosting. Computer Speech and Language 16(3-4) (2002)

24. Walker, M.A., Stent, A., Mairesse, F., Prasad, R.: Individual and domain adaptation in sentence planning for dialogue. Journal of Artificial Intelligence Research (JAIR) 30, 413-456 (2007)

25. Wardrip-Fruin, N.: Expressive Processing: Digital fictions, computer games, and software studies. The MIT Press (2009) 